\title{
CORRECTION
}

Florian MülleriD • Malte Krack

\section{Correction to: Explanation of the self-adaptive dynamics of a harmonically forced beam with a sliding mass}

Published online: 14 October 2020

(C) Springer-Verlag GmbH Germany, part of Springer Nature 2020

\author{
Correction to: Arch Appl Mech (2020) 90:1569-1582 \\ https://doi.org/10.1007/s00419-020-01684-5
}

The authors regret to have made an implementation error in their simulation code. More specifically, the harmonic base excitation had a wrong sign, which made it inconsistent with the contact kinematics. In the following, we show that all of the simulated results of the original paper can be reproduced qualitatively. Hereby, it is inevitable to slightly adjust some of the system parameters. Precisely, the excitation parameters as well as the slider's gap size and in one case the slider's mass are adjusted. Original and corrected values are given in the figures' captions. The need to slightly change some of the unknown parameters appears plausible due to the very strong sensitivity of the self-adaptive process observed in previous experimental and numerical studies. As all results remain qualitatively the same, the entire text of the original papers, including description, interpretation and conclusions, is in no way affected by this error.
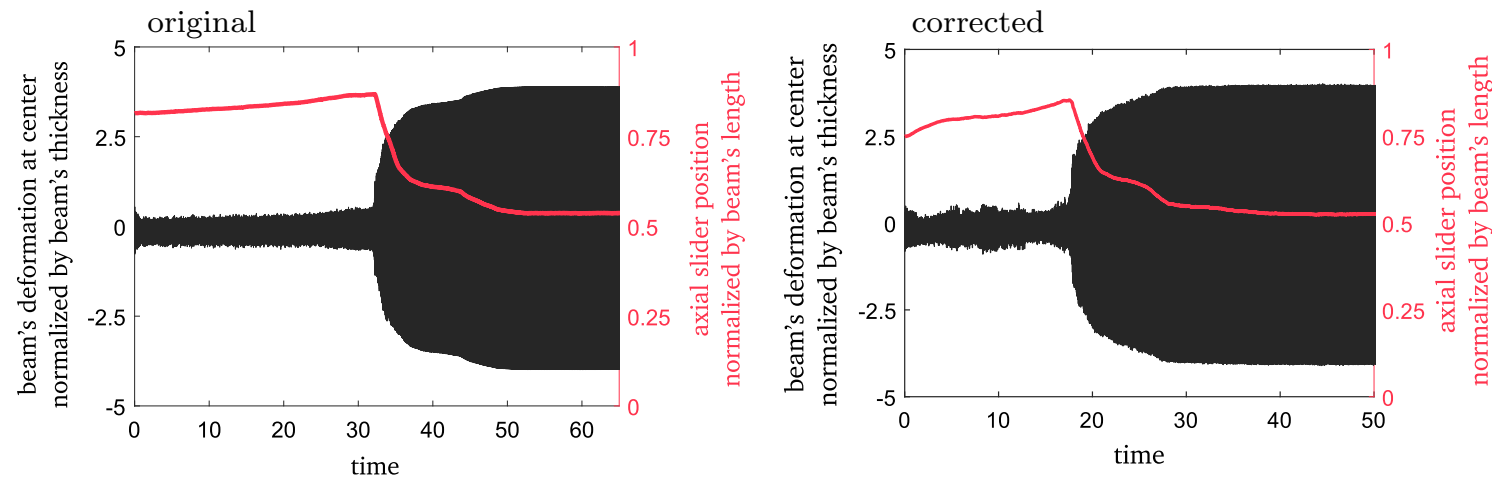

Fig. 1 Adjusted parameters: Original: $R / h=1.05$. Corrected: $R / h=1.1$.

The original article can be found online at https://doi.org/10.1007/s00419-020-01684-5.

F. Müller $(\varangle) \cdot$ M. Krack

Institute of Aircraft Propulsion Systems, University of Stuttgart, Pfaffenwaldring 6, 70569 Stuttgart, Germany

E-mail: mueller@ila.uni-stuttgart.de 

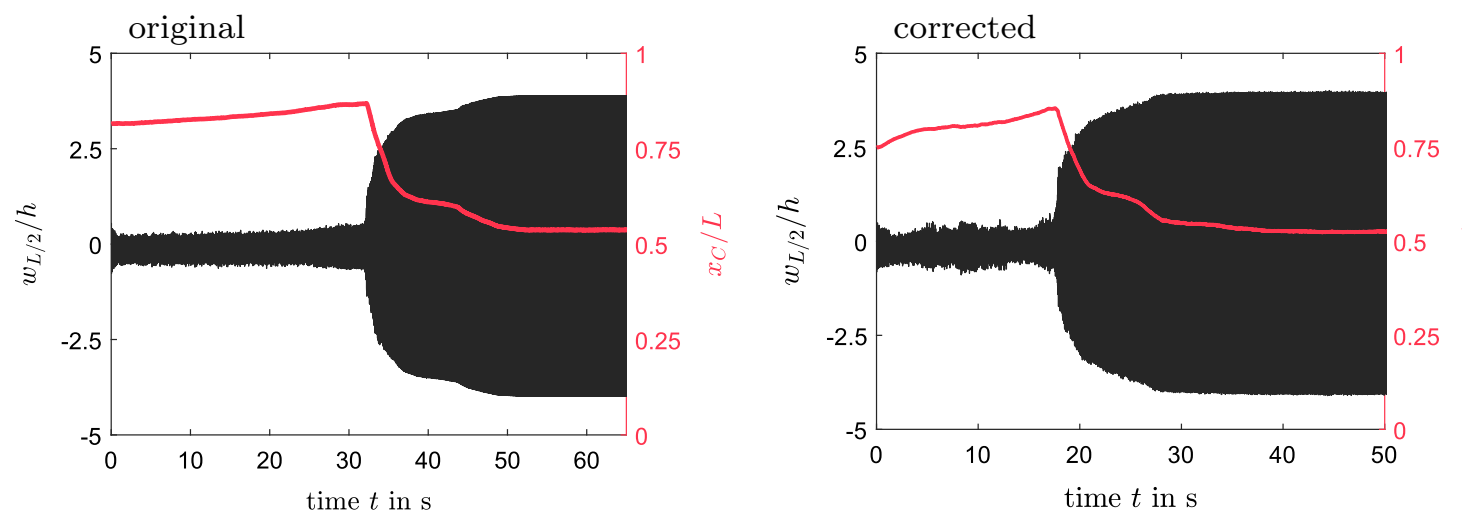

Fig. 5 Adjusted parameters: Original: $R / h=1.05$. Corrected: $R / h=1.1$.
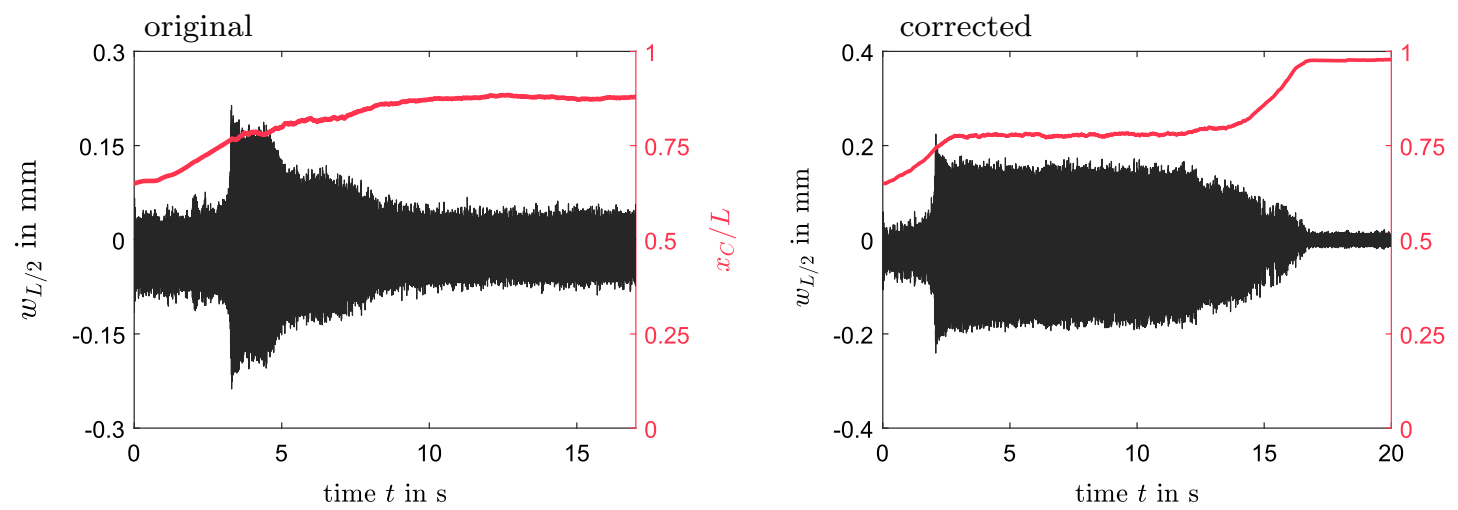

Fig. 6 Adjusted parameters: Original: $f_{\mathrm{ex}}=130 \mathrm{~Hz}, \hat{\ddot{w}}_{0}=20 \mathrm{~m} / \mathrm{s}^{2}, R / h=1.2$. Corrected: $f_{\text {ex }}=120 \mathrm{~Hz}, \hat{\ddot{w}}_{0}=10 \mathrm{~m} / \mathrm{s}^{2}, R / h=1.3$. Some differences are observed between original and corrected version, where the corrected one may fit the experiment better (see original paper).
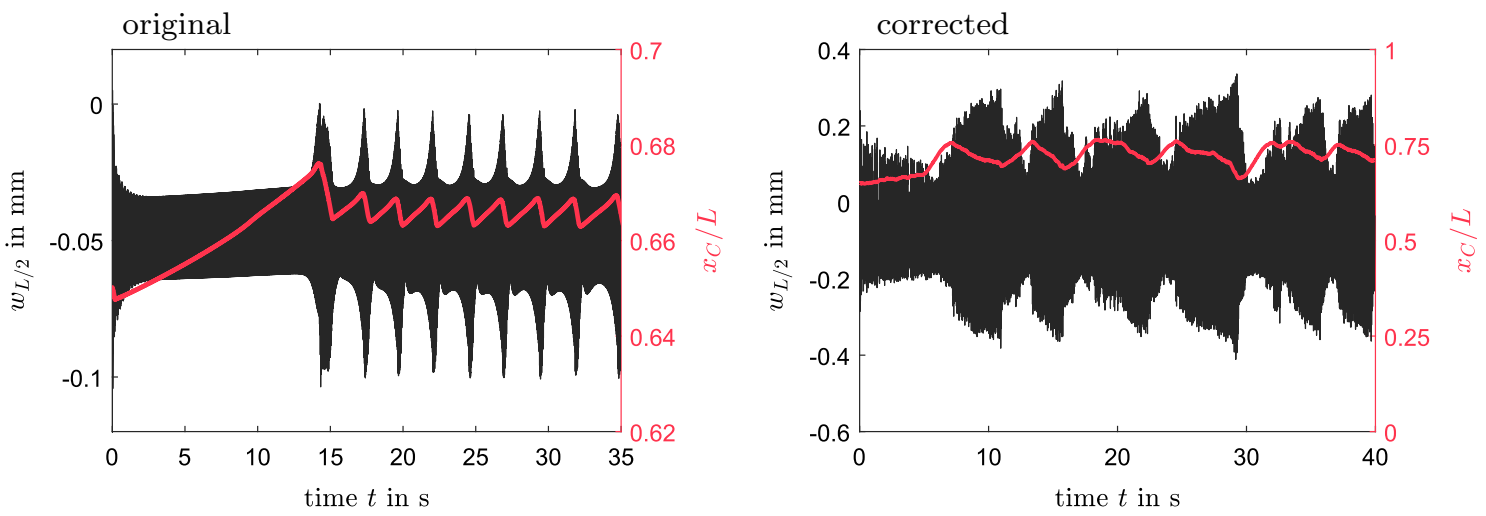

Fig. 7 Adjusted parameters: Original: $f_{\text {ex }}=150 \mathrm{~Hz}, \hat{\ddot{w}}_{0}=10 \mathrm{~m} / \mathrm{s}^{2}, R / h=1.1, \mathrm{~m} / \mathrm{m}_{\text {ref }}=$ $J_{y y}^{(C)} / J_{y y, \text { ref }}^{(C)}=2$. Corrected: $f_{\mathrm{ex}}=101 \mathrm{~Hz}, \hat{\tilde{w}}_{0}=19 \mathrm{~m} / \mathrm{s}^{2}, R / h=1.3, \mathrm{~m} / \mathrm{m}_{\mathrm{ref}}=$ $J_{y y}^{(C)} / J_{y y, \text { ref }}^{(C)}=4.8$. 
original

(a) reference

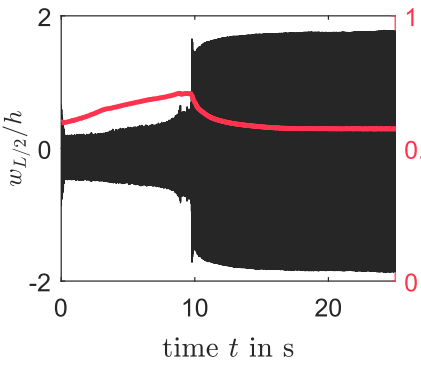

corrected

(a) reference

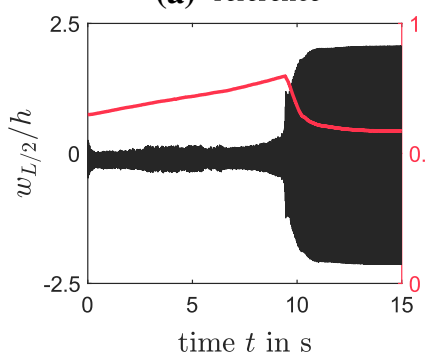

(b) linear beam

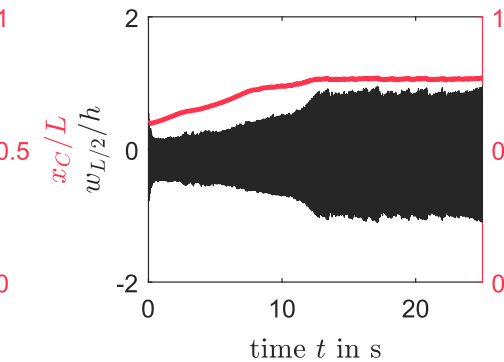

(c) no friction

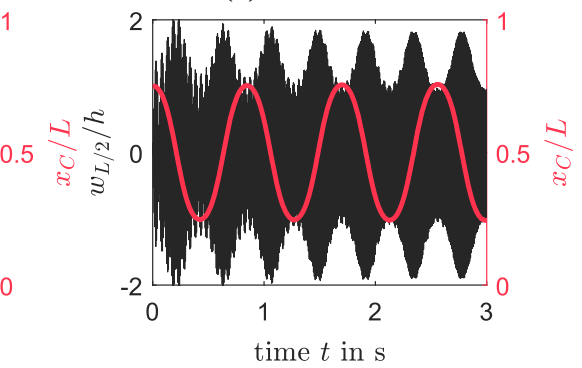

Fig. 8 Adjusted parameters: Original: $f_{\mathrm{ex}}=130 \mathrm{~Hz}, \hat{\ddot{w}}_{0}=30 \mathrm{~m} / \mathrm{s}^{2}$. Corrected: $f_{\mathrm{ex}}=150 \mathrm{~Hz}$, $\hat{\ddot{w}}_{0}=12.5 \mathrm{~m} / \mathrm{s}^{2}$.

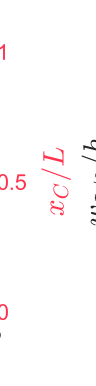

(b) linear beam

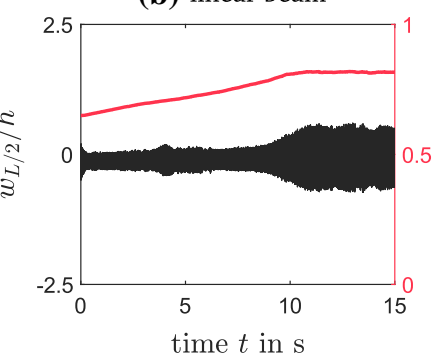

(c) no friction

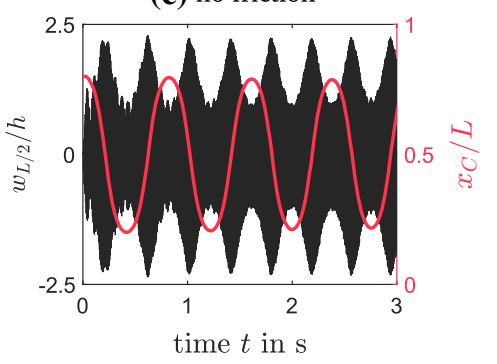



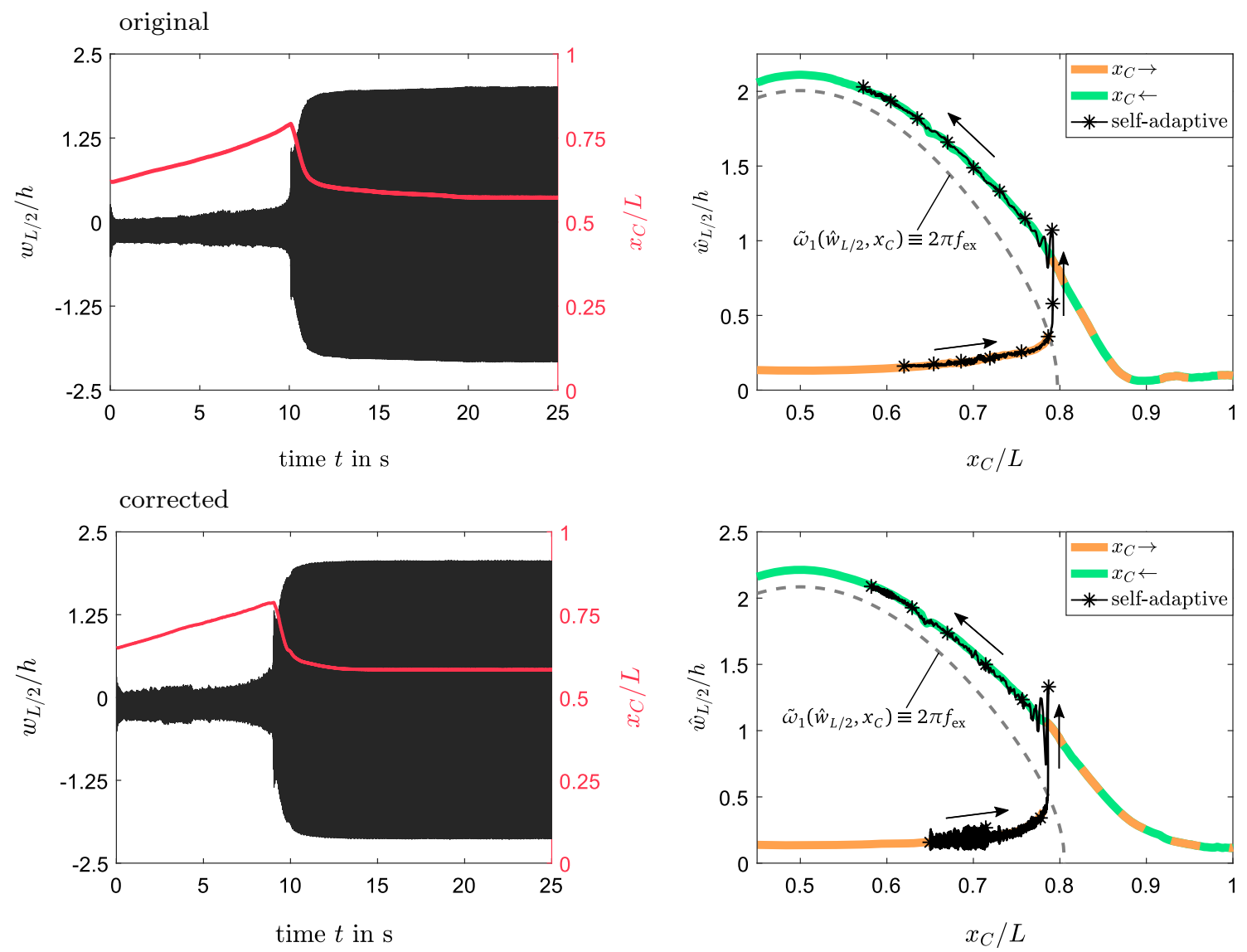

Fig. 10 Adjusted parameters: Original: $f_{\mathrm{ex}}=145 \mathrm{~Hz}, \hat{\ddot{w}}_{0}=20 \mathrm{~m} / \mathrm{s}^{2}$. Corrected: $f_{\mathrm{ex}}=$ $149 \mathrm{~Hz}, \hat{\ddot{w}}_{0}=15 \mathrm{~m} / \mathrm{s}^{2}$. 

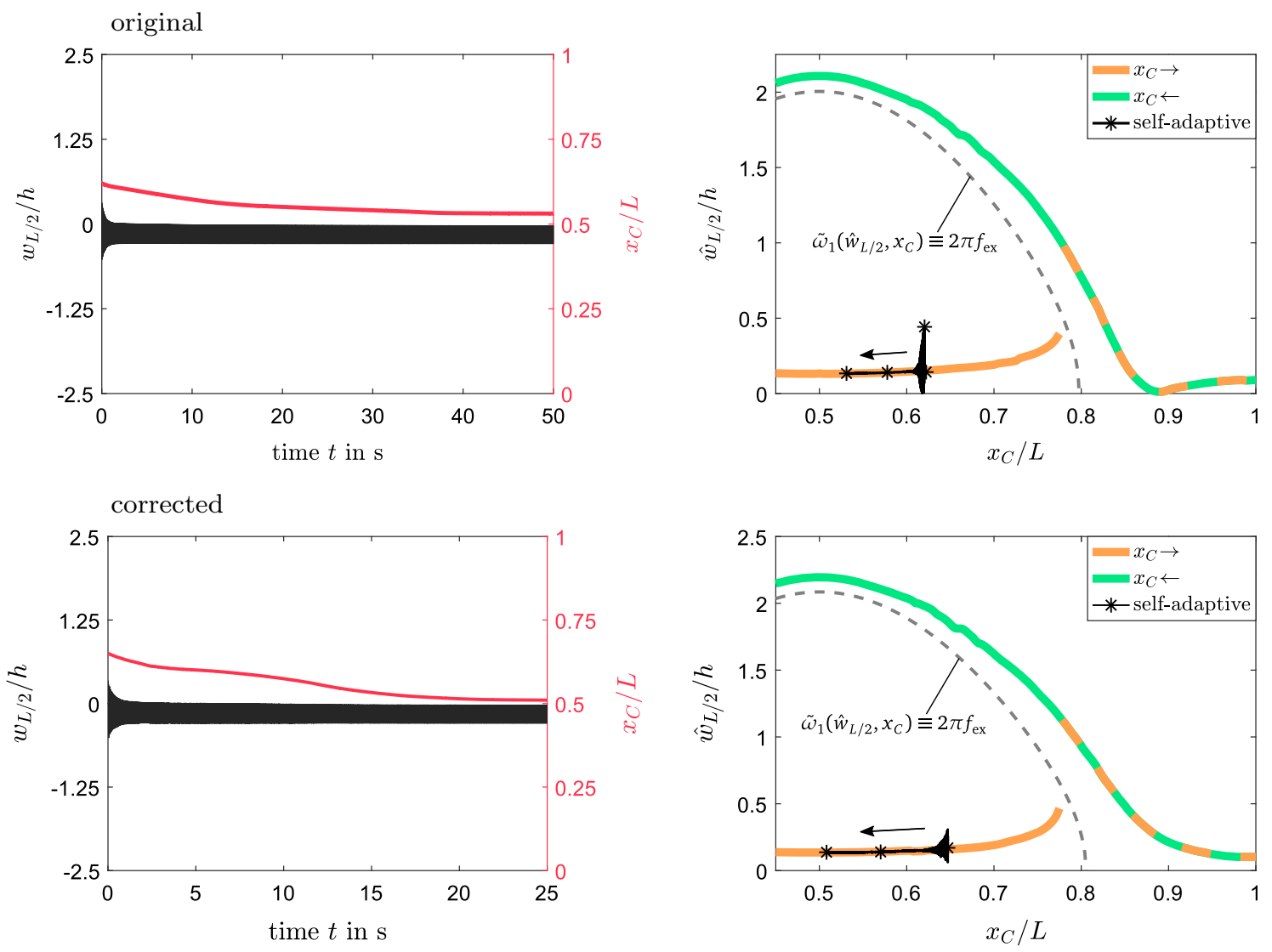

Fig. 11 Adjusted parameters: Original: $f_{\mathrm{ex}}=145 \mathrm{~Hz}, \hat{\ddot{w}}_{0}=20 \mathrm{~m} / \mathrm{s}^{2}$. Corrected: $f_{\mathrm{ex}}=$ $149 \mathrm{~Hz}, \hat{\ddot{w}}_{0}=15 \mathrm{~m} / \mathrm{s}^{2}$. 

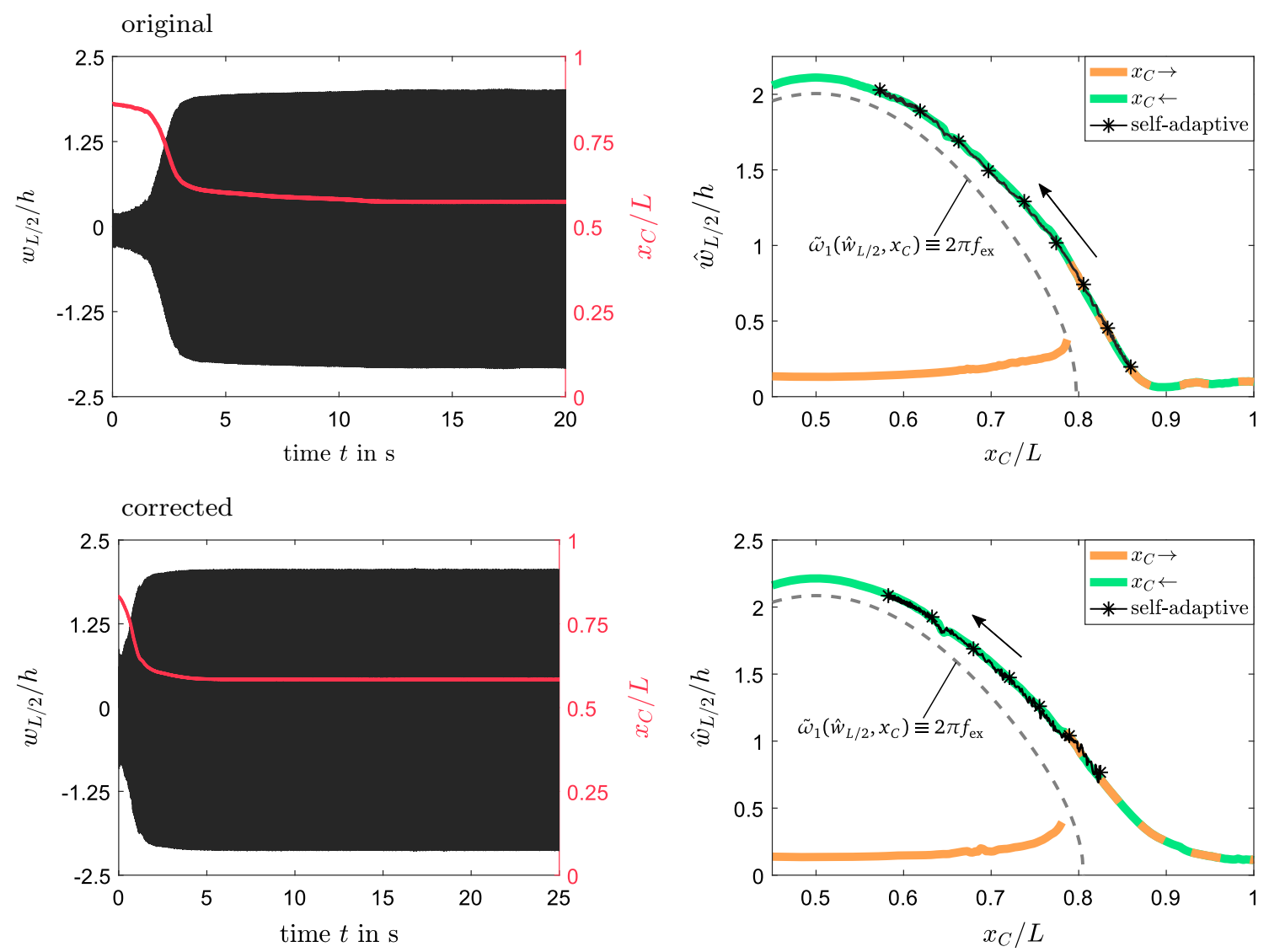

Fig. 12 Adjusted parameters: Original: $f_{\mathrm{ex}}=145 \mathrm{~Hz}, \hat{\ddot{w}}_{0}=20 \mathrm{~m} / \mathrm{s}^{2}$. Corrected: $f_{\mathrm{ex}}=$ $149 \mathrm{~Hz}, \hat{\ddot{w}}_{0}=15 \mathrm{~m} / \mathrm{s}^{2}$. 

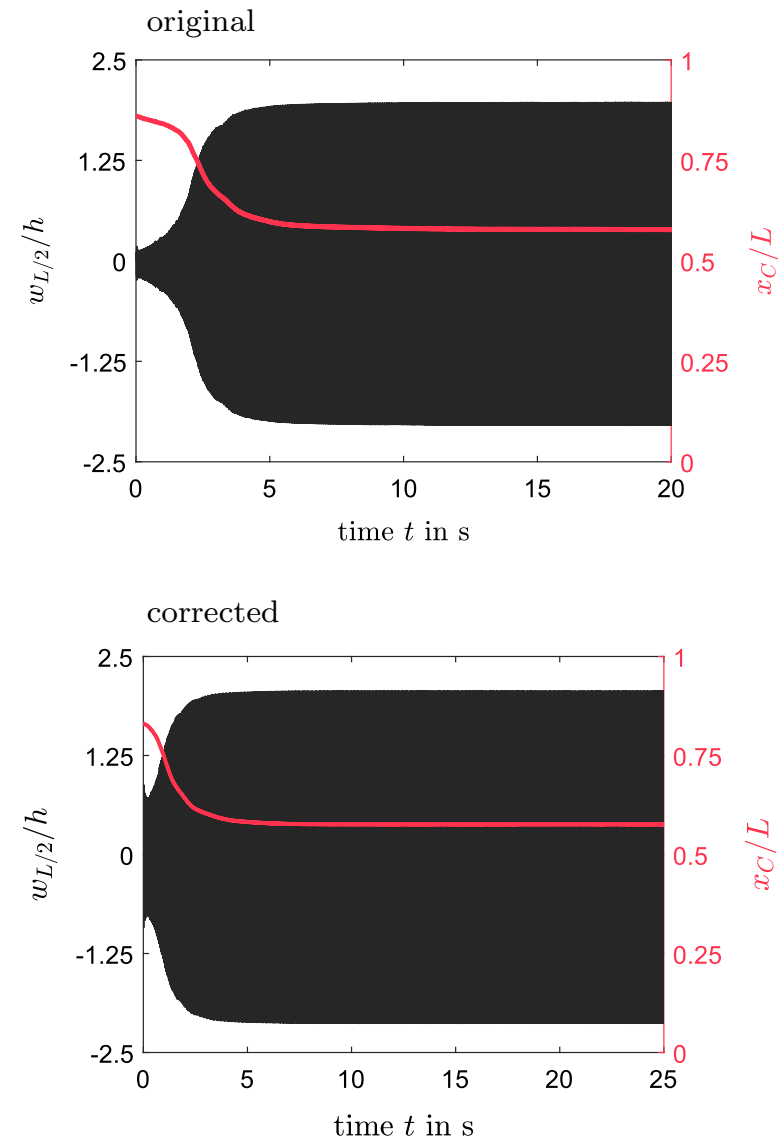
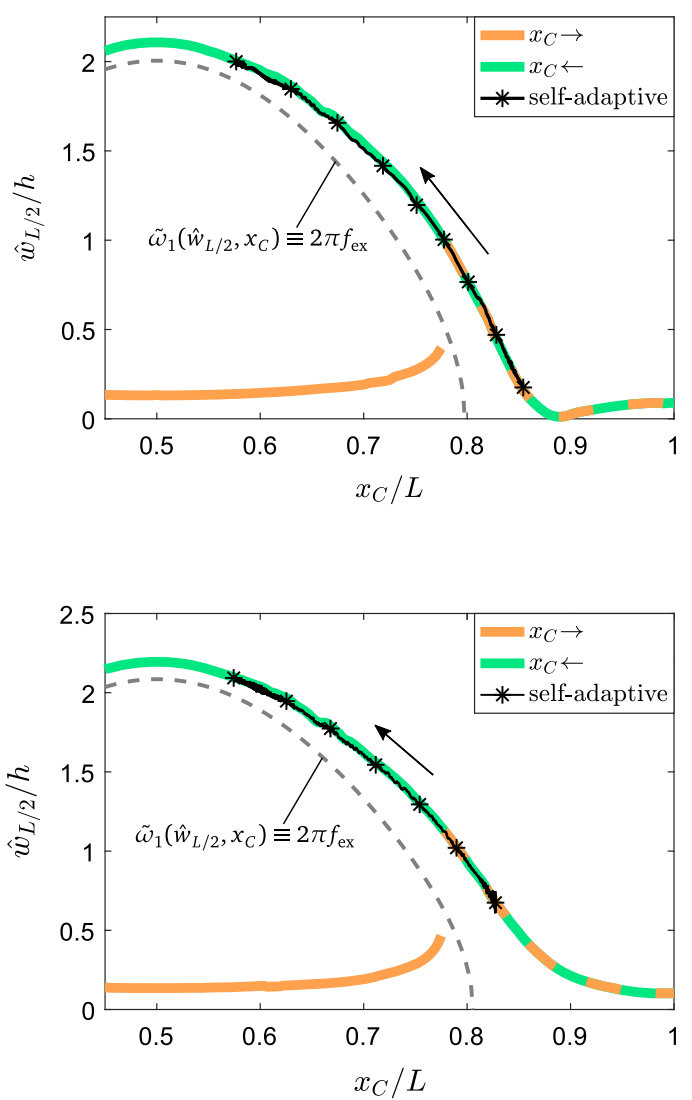

Fig. 13 Adjusted parameters: Original: $f_{\mathrm{ex}}=145 \mathrm{~Hz}, \hat{\vec{w}}_{0}=20 \mathrm{~m} / \mathrm{s}^{2}$. Corrected: $f_{\mathrm{ex}}=$ $149 \mathrm{~Hz}, \hat{\tilde{w}}_{0}=15 \mathrm{~m} / \mathrm{s}^{2}$. 

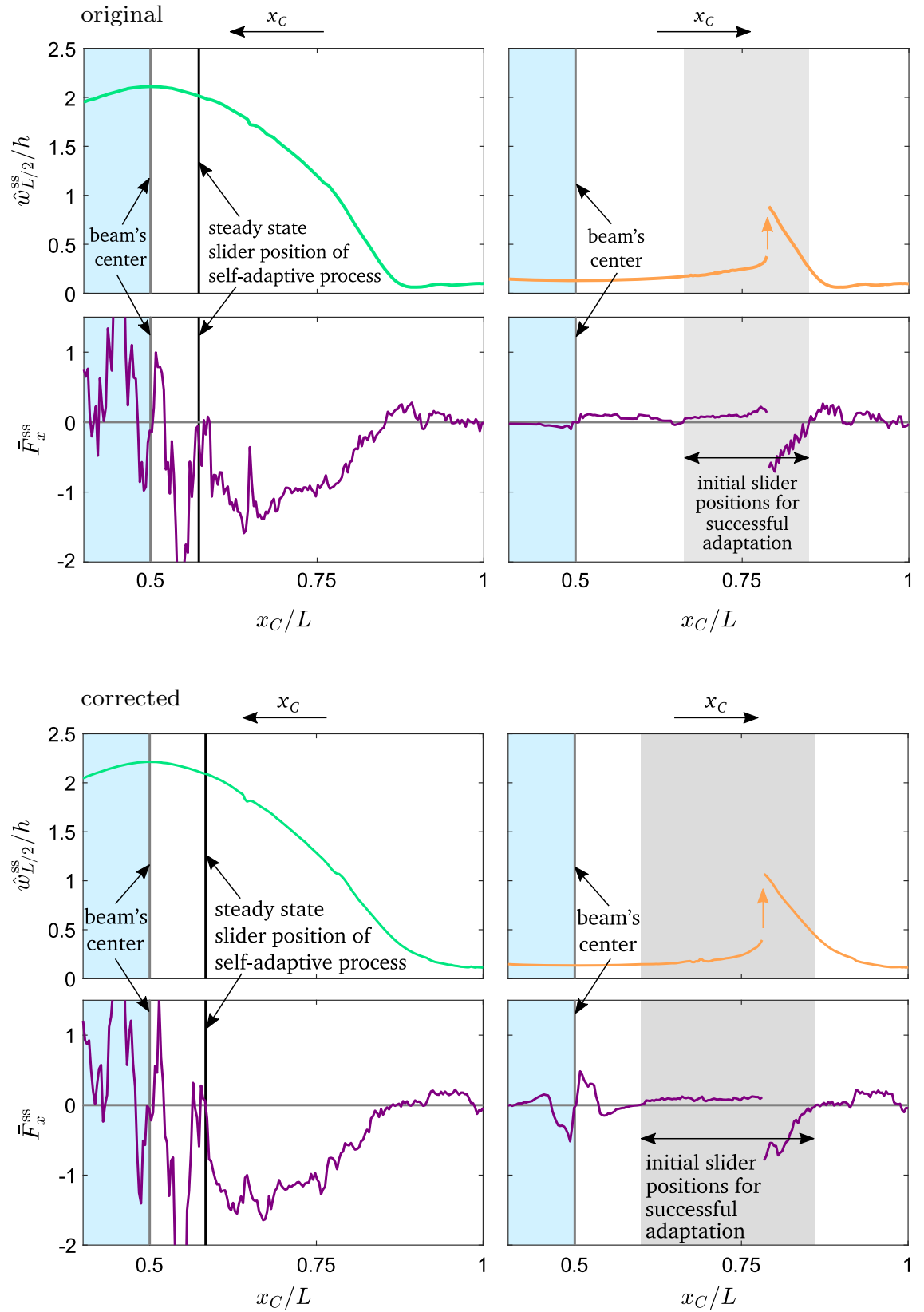

Fig. 14 Adjusted parameters: Original: $f_{\mathrm{ex}}=145 \mathrm{~Hz}, \hat{\ddot{w}}_{0}=20 \mathrm{~m} / \mathrm{s}^{2}$. Corrected: $f_{\mathrm{ex}}=$ $149 \mathrm{~Hz}, \hat{\ddot{w}}_{0}=15 \mathrm{~m} / \mathrm{s}^{2}$.

Publisher's Note Springer Nature remains neutral with regard to jurisdictional claims in published maps and institutional affiliations. 